\title{
Climate information based streamflow and rainfall forecasts for Huai River basin using hierarchical Bayesian modeling
}

\author{
X. Chen ${ }^{1,6}$, Z. Hao ${ }^{1}$, N. Devineni ${ }^{2,3}$, and U. Lall ${ }^{4,5}$ \\ ${ }^{1}$ State Key Laboratory of Hydrology-Water Resources and Hydraulic Engineering, Hohai University, Nanjing 210098, China \\ ${ }^{2}$ Department of Civil Engineering, The City University of New York (City College), New York, NY 10031, USA \\ ${ }^{3}$ NOAA-Cooperative Remote Sensing Science and Technology Center, The City University of New York (City College), \\ New York, NY 10031, USA \\ ${ }^{4}$ Columbia Water Center, The Earth Institute, Columbia University, New York, NY 10027, USA \\ ${ }^{5}$ Department of Earth and Environmental Engineering, Columbia University, New York, NY 10027, USA \\ ${ }^{6}$ Bureau of Hydrology, Changjiang Water Resources Commission, Wuhan 430010, China
}

Correspondence to: N. Devineni (ndevineni@ ccny.cuny.edu)

Received: 22 July 2013 - Published in Hydrol. Earth Syst. Sci. Discuss.: 12 September 2013

Revised: 8 March 2014 - Accepted: 14 March 2014 - Published: 29 April 2014

\begin{abstract}
A Hierarchal Bayesian model is presented for one season-ahead forecasts of summer rainfall and streamflow using exogenous climate variables for east central China. The model provides estimates of the posterior forecasted probability distribution for 12 rainfall and 2 streamflow stations considering parameter uncertainty, and cross-site correlation. The model has a multi-level structure with regression coefficients modeled from a common multi-variate normal distribution resulting in partial pooling of information across multiple stations and better representation of parameter and posterior distribution uncertainty. Covariance structure of the residuals across stations is explicitly modeled. Model performance is tested under leave-10-out cross-validation. Frequentist and Bayesian performance metrics used include receiver operating characteristic, reduction of error, coefficient of efficiency, rank probability skill scores, and coverage by posterior credible intervals. The ability of the model to reliably forecast season-ahead regional summer rainfall and streamflow offers potential for developing adaptive water risk management strategies.
\end{abstract}

\section{Introduction}

The Huai River basin (Fig. 1), located between the Yangtze and Yellow River basins is the most densely inhabited river basin and the main cropping area in China. The total drainage area of $270000 \mathrm{~km}^{2}$ is divided into the Huai River catchment $\left(190000 \mathrm{~km}^{2}\right)$ and the Yishusi River catchment $\left(80000 \mathrm{~km}^{2}\right)$ by a paleo-channel of Yellow River. The mean annual precipitation for the Huai River basin is approximately $900 \mathrm{~mm}$, of which 50-75\% occurs during the summer monsoon season. There are 36 large reservoirs in the basin primarily designed for water supply and flood control (Zhang et al., 2012). Natural climate variations in conjunction with increasing population demands are causing severe water stress in the region. Moreover, the region is susceptible to droughts and floods with a recurrent frequency of four years on average (Yan et al., 2013; Cheng et al., 2012). Such variations in water supply are often related to inter-annual fluctuations in large-scale climatic patterns. In this paper, potential climate teleconnections are explored and formalized into a predictive model in a Bayesian framework which allows for formal uncertainty reduction and modeling. Applications for water, food and energy management using such probabilistic forecasts could be developed as part of a strategy for climate risk mitigation and for adaptation to a variable climate.

Interest in the development and application of long-lead hydrologic forecasts has grown over the last decade primarily because of the improved monitoring of sea surface temperature (SST) in the tropical Pacific and advances in experimental climate forecasts from general circulation models (GCMs). Since GCM-predicted fields are usually available at large spatial scales, one needs to apply either dynamical or 


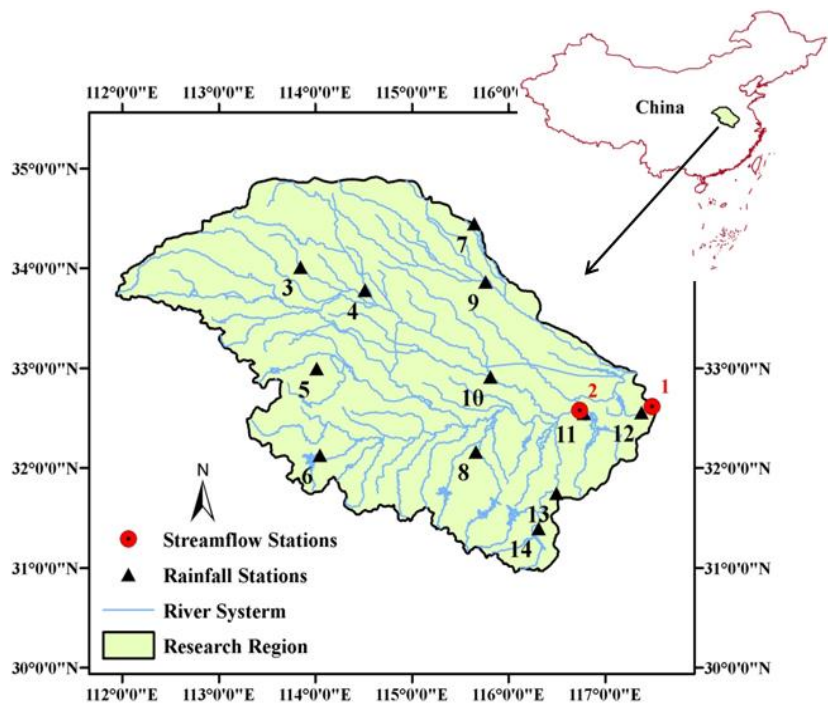

Fig. 1. Location of the study area.

statistical downscaling to develop regional hydrologic forecasts (Robertson et al., 2004; Gangopadhyay et al., 2005). Alternately, one can develop a low-dimensional statistical model by relating the observed rainfall or streamflow to identified climatic precursors (e.g., El Ninõ-Southern Oscillation (ENSO) indices) for the given site (Souza and Lall, 2003). A key aspect in developing such dynamic or statistical models is the ability to accurately represent the uncertainties, both at the model representation level and at the parameter estimation level. Developing statistical schemes that can address simultaneous prediction at multiple sites and for multiple variables while addressing their correlation structure is often a challenge.

Hierarchical Bayesian methods provide the opportunity to explicitly quantify the parameter uncertainty through each estimation stage using appropriate conditional and prior distributions. This allows a better representation of model and parameter uncertainties. Recently, Devineni et al. (2013) presented a hierarchical Bayesian regression strategy for estimating streamflow at multiple locations using various model structures to pool information across multiple sites to an appropriate degree such that the features that are common to the site regression and those that vary across sites can be identified for an overall reduction in parameter uncertainty while preserving the structure in errors across the stations. Here, a similar Hierarchical Bayesian approach is developed for regional rainfall and streamflow forecasts using appropriate climate indicators that could be derived from GCMs or observed climate fields. The application focuses on the upper and middle regions of the Huai River basin which are of interest for local management, and may have similar climatic forcing. Section 2 provides a brief description of the study area, data sources and the climate predictor identification procedure. The hierarchical Bayesian regression model is presented in Sect. 3. In Sect. 4, the cross-validated results are presented. A summary is finally presented.

\section{Data description}

\subsection{Streamflow and rainfall data}

We used streamflow data from two stations and rainfall data from 12 stations in this study to develop regional hydrologic forecasts. The streamflow data are from the Bengbu $\left(117.38^{\circ} \mathrm{E}, 32.56^{\circ} \mathrm{N}\right)$ and the Lutaizi $\left(116.79^{\circ} \mathrm{E}, 32.57^{\circ} \mathrm{N}\right)$ hydrological stations (shown as red dots in Fig. 1). Twelve rainfall stations with at least 50 years of data were selected in the contributing section of the basin (shown as filled triangles in Fig. 1). The details of the hydrologic stations including the number of years of data records are shown in Table 1. Preliminary analysis of the seasonality of streamflow and area averaged rainfall show that more than $50 \%$ of the annual rainfall and streamflow occurs in June-July-August (JJA) (Fig. 2). Consequently, a prediction of the summer monsoon rainfall and streamflow in June or earlier is of interest.

\subsection{Climate teleconnection and predictor identification}

Xu et al. (2007) and Kwon et al. (2009) developed seasonahead streamflow forecasts for the Yangtze River on the Three Gorges Dam using exogenous climate indices from eastern Indian Ocean and western Pacific Ocean. Recently, Liu et al. (2013) and Linderhorm et al. (2013) investigated the relation between East Asian monsoon rainfall and North Atlantic sea surface temperature conditions using observations and paleo-reconstructed records. So far little work has been done for climate informed hydrologic prediction for the Huai River basin. To identify predictors that influence the regional hydroclimate in the basin during JJA season, we consider SST anomaly conditions during FebruaryMarch-April (FMA, 3 months lag) and October-NovemberDecember (OND, 6 months lag) obtained from the Hadley Center SST dataset (HADSST2) (Rayner et al., 2006). Figure 3 shows the Spearman's rank correlation between the observed streamflow during JJA at the Bengbu hydrologic station and the pre-season SST conditions. The 3-month lag correlation (i.e., JJA streamflow with FMA SSTa) and the 6month lag correlation (i.e., JJA streamflow with OND SSTa) are shown in Fig. 3a and b, respectively. From Fig. 3a, we see that the SST1 region $\left(155-175^{\circ} \mathrm{E}\right.$ and $\left.40-50^{\circ} \mathrm{N}\right)$ correlates with the summer streamflow in the Huai River basin. This region is associated with the Kuroshio current which is the west side of the clockwise North Pacific Ocean gyre. This phenomenon was previously identified by Geng et al. (1997). The warmer SSTs in the Kuroshio current region and the mid-latitude central Pacific in late spring can generate a large-scale atmospheric circulation pattern over the Asia-Pacific region that is favorable for precipitation in the North China region. Warm conditions in this region result in 

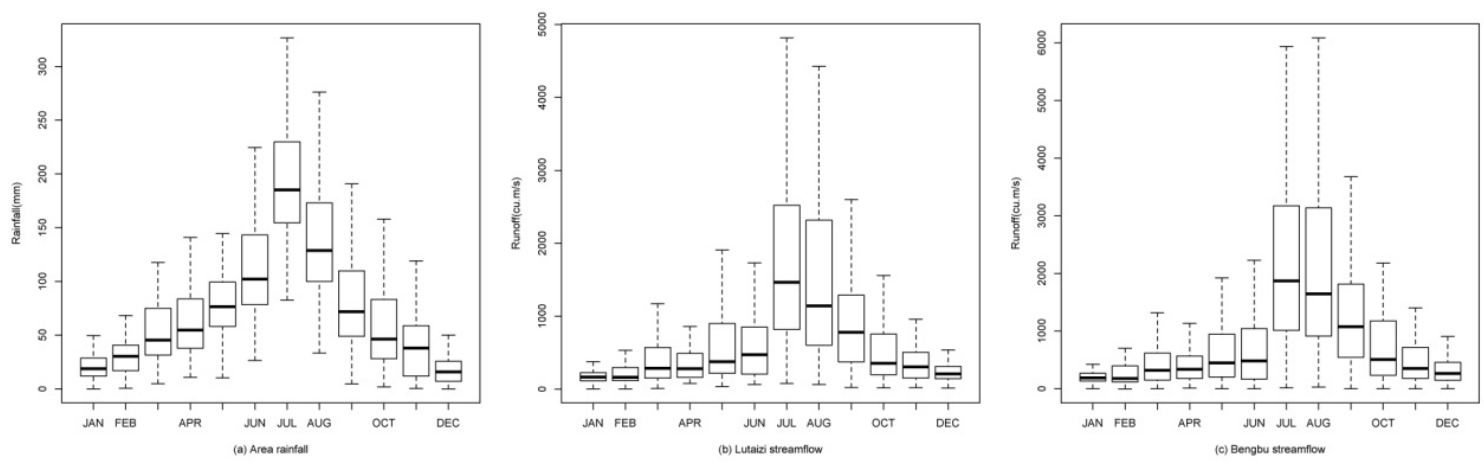

Fig. 2. Seasonality of area rainfall and streamflow in study region.

Table 1. Detail information for streamflow and rainfall station used in this study.

\begin{tabular}{|c|c|c|c|c|c|}
\hline $\begin{array}{l}\text { Station } \\
\text { ID }\end{array}$ & $\begin{array}{l}\text { Station } \\
\text { name }\end{array}$ & Abbreviation & $\begin{array}{r}\text { Elevation } \\
(\mathrm{m})\end{array}$ & Category & $\begin{array}{c}\text { Actual data record } \\
\& \text { used for reconstruction }\end{array}$ \\
\hline 1 & Bengbu & $\mathrm{BB}$ & 10.0 & streamflow & 1951-2010 \\
\hline 2 & Lutaizi & LTZ & 19.0 & streamflow & $1951-2010$ \\
\hline 3 & Xuchang & $\mathrm{XC}$ & 66.8 & rainfall & $1952-2010$ \\
\hline 4 & Xihua & XH & 52.6 & rainfall & 1955-2010 \\
\hline 5 & Zhumadian & ZMD & 82.7 & rainfall & $1958-2010$ \\
\hline 6 & Xinyang & XY & 114.5 & rainfall & $1951-2010$ \\
\hline 7 & Shangqiu & SQ & 50.1 & rainfall & 1953-2010 \\
\hline 8 & Gushi & GS & 57.1 & rainfall & 1952-2010 \\
\hline 9 & Bozhou & $\mathrm{BZ}$ & 37.7 & rainfall & 1953-2010 \\
\hline 10 & Fuyang & FY & 30.6 & rainfall & $1953-2010$ \\
\hline 11 & Shouxian & SX & 22.7 & rainfall & 1955-2010 \\
\hline 12 & Bengbu & BB & 18.7 & rainfall & $1952-2010$ \\
\hline 13 & Liuan & LA & 60.5 & rainfall & 1956-2010 \\
\hline 14 & Huoshan & HS & 68.1 & rainfall & 1954-2010 \\
\hline
\end{tabular}

above-normal inflow conditions in the Huai River. Similarly, from Fig. 3b, we can see that 6-month prior conditions in the North Atlantic Ocean identified as SST2 $\left(15^{\circ} \mathrm{W}-5^{\circ} \mathrm{E}\right.$ and $35-55^{\circ} \mathrm{N}$ ) influence the summer flows in the basin. This is in line with an earlier finding (Gu et al., 2009a) which showed that the East Asian summer monsoons are strongly related to a tripole mode of the North Atlantic SST anomalies in the preceding winter that typically enhances the stationary wave-train propagating from west Eurasia to East Asia. The correlations with SST1 and SST2 are statistically significant at the $95 \%$ level.

In addition to the SST anomaly conditions, we also considered the 4-month lagged (February-March-April) North Atlantic oscillations (NAO) (Hurrell et al., 2003), summer North Atlantic oscillation (SNAO) (Folland et al., 2009) and 6-month lagged (November-December-January) Atlantic Multi-decadal Oscillation (AMO) (Knight et al., 2006) as candidate predictors. Gu et al. (2009b) showed that the January and March NAO modulates the summer rainfall patterns over China. Following Linderholm et al. (2011), we selected the SNAO as one of the predictors as it influences the storm tracks over China with wetter than normal conditions during positive SNAO phase and drier than normal conditions during negative phase. Similarly, AMO has been shown as an important covariate for climate in central Asia with positive AMO phase leading to strong southeast summer monsoons and late retrieval (Lu et al., 2006). We also checked the relationship between ENSO indices, Pacific decadal oscillation and the Northern Hemisphere snow cover with summer streamflow and rainfall in the basin. There was no statistically significant correlation between these covariates and streamflow or rainfall in the region. Table 2 summarizes the correlations between the two streamflow stations, area averaged rainfall and the climate predictors selected for the study.

\section{Methodology}

\subsection{Hierarchical Bayesian model}

In this paper, we follow an approach similar to that used by Devineni et al. (2013) for tree-ring-based streamflow reconstruction, but for multi-variate seasonal forecasts for regional 
Table 2. The correlation between streamflow/area rainfall and climate predictors chosen in this study*.

\begin{tabular}{lccccc}
\hline & SST1 & SST2 & AMO & NAO & SNAO \\
\hline Lutaizi & 0.44 (FMA) & 0.45 (OND) & 0.28 (NDJ) & -0.26 (FMA) & 0.39 (FMA) \\
Bengbu & 0.44 (FMA) & 0.47 (OND) & 0.21 (NDJ) & -0.22 (FMA) & 0.34 (FMA) \\
Area rainfall & 0.46 (FMA) & 0.51 (OND) & 0.36 (NDJ) & -0.27 (FMA) & 0.36 (FMA) \\
\hline
\end{tabular}

* () is the selected period of the predictor for streamflow prediction; Area rainfall is the average rainfall of 12 stations in the study region.
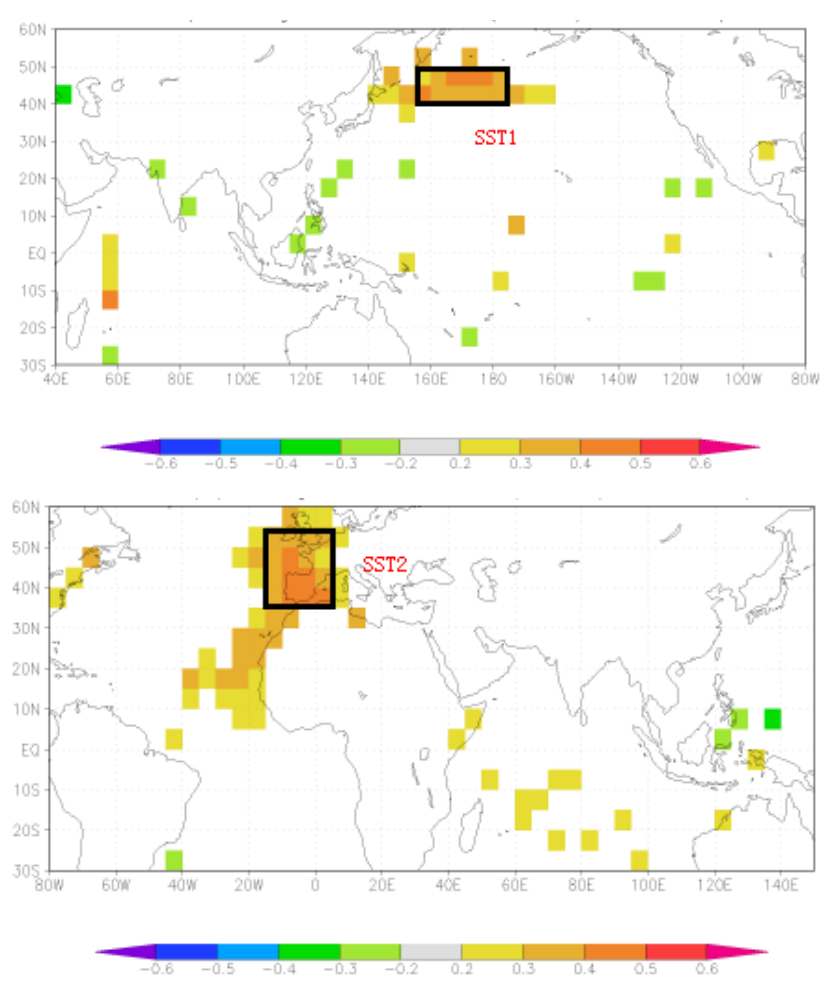

Fig. 3. SST regions (SST1 and SST2) that influence the rainfall and streamflow in the Huai River basin. SST regions that have significant correlation at $95 \%$ confidence interval $(>0.25$ or $<-0.25)$ are considered as predictors for the hierarchical Bayesian model. (SST1: $40-50^{\circ} \mathrm{N}, 155-175^{\circ} \mathrm{E}$; SST2: $35-55^{\circ} \mathrm{N}, 15^{\circ} \mathrm{W}-5^{\circ} \mathrm{E}$ ).

streamflow and rainfall. The basic idea is that a particular climate predictor may inform the rainfall or streamflow anomaly at each of the sites in the region in a similar way. If the response were exactly the same, predicting the average of the station values or pooling all the data into the same regression would be effective since that would reduce the uncertainty associated with parameter estimation. However, the response across the rainfall and the streamflow stations may vary systematically due to local conditions or averaging scale (e.g., for a large river basin vs. small or point rainfall). The hierarchical model can be used for partial pooling of this common information, by considering multiple levels of modeling. The individual regression coefficients for each site on each climate predictor are estimated at the first level.
The second level estimates the average regression coefficient across sites and its variance, thus allowing variation in the response across sites, but also its potential shrinkage to an appropriate degree through an estimation of the variance. If the variance estimated is large, then the model tends towards a model that would be formed if each site was regressed independently on the predictor. If the variance is small, then the model tends towards a fully pooled regression model, and the responses are deemed homogeneous. Partial pooling reduces the equivalent number of independent parameters, resulting in lower uncertainty in parameters estimates, and therefore reduced uncertainty in the final forecasts. The general modeling framework is presented as follows.

Given that the station rainfall at location $i$ for year $t$ is $R_{i t}$, and station streamflow at location $i$ for year $t$ is $S_{i t}$, we form $\mathbf{Y}=\{\mathbf{R}, \mathbf{S}\}$. The streamflow and rainfall data $\mathbf{Y}$ are assumed to come from a distribution (process model) with probability density function $f(\mathbf{Y} \mid \boldsymbol{\theta})$, where $\boldsymbol{\theta}$ is a parameter vector. In the application presented here, we consider that $\log (\mathbf{Y})$ is normally distributed. This assumption was checked using the Shapiro-Wilk test and Kolmogorov-Smirnov test on the logtransformed data. Analysis of the residuals using the quantile plots and normality tests also showed that the normal assumption of the log-transformed flows is valid. Where a linear model is considered for $\log (\mathbf{Y})$ in terms of a set of climate predictors, the regression coefficients are interpretable as the fractional change in $\mathbf{Y}$ given the corresponding predictor. This allows a consideration of partial pooling of response to a particular climate predictor across rainfall and streamflow data series that may have a disparate range or scale of values. The first level of the model considers that at each site $i$, $\log \left(Y_{i}\right)$ is described by a normal distribution with time varying mean $\mu_{i t}$ that is informed by a regression on the five predictors $\mathbf{X}_{t}$ with intercepts $\alpha_{i}$ and a $(5 \times 14)$ regression coefficient matrix $\boldsymbol{\beta}$. The "errors" from the regression model are considered to be spatially correlated with a $(14 \times 14)$ covariance matrix $\boldsymbol{\Sigma}$. The second level of the model considers that the regression coefficient matrix $\boldsymbol{\beta}$ can be modeled as coming from a multi-variate normal distribution with a $(5 \times 1)$ mean vector $\boldsymbol{\mu}_{\beta}$ which represents the average regression coefficient across the 14 sites for each predictor, and a $(5 \times 5)$ covariance matrix $\Sigma_{\beta}$ that takes into account the correlation across the predictors associated with the common effect across the 14 sites. The $\boldsymbol{\mu}_{\beta}$ and $\boldsymbol{\Sigma}_{\beta}$ are called hyperparameters. The model 
and the priors associated with the parameters and the hyperparameters are presented below:

Level 1: $\log \left(\mathbf{Y}_{t}\right) \sim N\left(\boldsymbol{\mu}_{t}, \boldsymbol{\Sigma}\right)$

$\mu_{i t}=\alpha_{i}+\mathbf{X}_{t} \boldsymbol{\beta}$

Level2: $\boldsymbol{\beta} \sim \operatorname{MVN}\left(\boldsymbol{\mu}_{\beta}, \boldsymbol{\Sigma}_{\beta}\right) \ldots$

With priors modeled as

$\alpha_{i} \sim N(0,10000)$

$\boldsymbol{\mu}_{\beta} \sim N(0,10000)$

$\Sigma_{\beta} \sim$ Inv-Wishart $_{v_{0}}\left(\Lambda_{0}\right)$

$\boldsymbol{\Sigma} \sim$ Inv-Wishart $_{v_{1}}\left(\Lambda_{1}\right)$

The prior for the covariance matrix $\boldsymbol{\Sigma}_{\beta}$ is taken to be the inverse Wishart distribution with a scale matrix $\Lambda_{0}$ and $\nu_{0}$ degrees of freedom. In our applications, the scale matrices $\Lambda_{0}$ and $\Lambda_{1}$ were specified as an identity matrix (I) and the degrees of freedom $v_{0}$ and $v_{1}$ were set to one more than the dimension of the matrix (i.e., the total number of predictors, five for $\boldsymbol{\Sigma}_{\beta}$ and total number stations, 14 for $\boldsymbol{\Sigma}$ ) to induce a uniform prior distribution on the variance (Gelman and Hill, 2007). This choice of priors was made for computational convenience and represents a simpler model than could be formulated if all parameter covariance were to be modeled. The joint posterior distribution $p(\boldsymbol{\theta} \mid$ data $)$, of the complete parameter vector $\boldsymbol{\theta}$ is derived by combining the prior distributions and the likelihood functions. The parameters $\boldsymbol{\theta}$ are estimated using WinBUGS (Spiegelhalter et al., 1996) which employs the Gibbs sampler, a Markov chain Monte Carlo (MCMC) method for simulating the posterior probability distribution of the parameters conditional on the current choice of parameters and the data. A discussion on such model constructs and their comparison to a no pooling model that estimates independent regressions across sites and to a full pooling model that ignores the cross-site variations in response is presented in Devineni et al. (2013). Several hydrologic applications using Bayesian model constructs have also been developed and demonstrated in Lima and Lall (2009, 2010), and Kwon et al. (2008). Renard et al. (2013) present a useful tutorial and examples of related Bayesian models for hydroclimatic applications.

\subsection{Cross-validation}

Cross-validation statistics computed over different blocks of data can reveal how well the Bayesian model can perform in truly out of sample predictions recognizing that different climate epochs may lead to different model fits and performance. We evaluate the model using an $\mathrm{m}$-fold crossvalidation technique. A sample is formed by leaving out $m$ randomly selected data points from the observational data set for validation and the Bayesian model is developed using the remaining $(n-m)$ observations. This process is repeated several times to obtain an ensemble of validation metrics resulting from each randomly selected model. In the applications presented in the later section, $m$ was $10, n$ was 50 , and 30 sample models were fit. We use three traditional performance metrics, reduction of error (RE) and coefficient of efficiency (CE) and the rank probability skill score (RPSS), as measures of model performance to compare the forecasted posterior mean and the distribution of the streamflow and rainfall estimates with the actual streamflow and rainfall data.

The reduction of error (RE) ranges from $-\infty$ to +1 and is similar to the $R^{2}$ statistic (Lorenz, 1956; Fritts, 1976).

$\mathrm{RE}=1.0-\frac{\sum_{t=1}^{n}\left(O_{t}-S_{t}\right)^{2}}{\sum_{t=1}^{n}\left(O_{t}-\overline{o_{\mathrm{c}}}\right)^{2}}$

In Eq. (5), $O_{t}$ and $S_{t}$ are the observed and the predicted posterior mean of the streamflow (transformed back to real space by taking anti-logs) in year $t$ of the validation period and $\bar{o}_{\mathrm{c}}$ is the mean of the observational data in the calibration period. $\mathrm{RE}>0$ indicates that the simulated streamflow contains useful information not contained in the calibration period. Similarly $\mathrm{RE}<0$ indicates that the simulations are poorer than climatology, i.e., the simulations are not better than the mean flows in the calibration period. The coefficient of efficiency (CE) is defined as

$\mathrm{CE}=1.0-\frac{\sum_{t=1}^{n}\left(O_{t}-S_{t}\right)^{2}}{\sum_{t=1}^{n}\left(O_{t}-\overline{o_{\mathrm{V}}}\right)^{2}}$.

In Eq. (6), $O_{t}$ and $S_{t}$ are the observed and the predicted posterior mean of the streamflow in year $t$ of the validation period and $\overline{o_{\mathrm{v}}}$ is the mean of the observational data in the validation period. $\mathrm{CE}<0$ indicates that the simulations are poorer than validation climatology, i.e., the simulations are not better than the mean flows in the validation period. $\mathrm{CE}$ is similar to RE, but used as a measure to evaluate the model under the validation period, it is a more rigorous metric.

In addition to RE and CE that measure the error in predicting the conditional mean, we also verify the RPSS to quantify the error in estimating the entire probability distribution of the forecast (Wilks, 2011; Candille and Talagrand, 2005; Gangopadhyay et al., 2005). The RPSS is based on the rank probability score (RPS) computed for each forecast and observation pair at each station in each year:

$\mathrm{RPS}=\sum_{\mathrm{k}=1}^{n}\left(S_{\mathrm{k}}-O_{\mathrm{k}}\right)^{2}$,

where $S_{\mathrm{k}}$ is the cumulative probability of the forecast for category k and $O_{\mathrm{k}}$ is the cumulative probability of the observation for category k. This is implemented as follows. First, the observed time series is used to develop 3 categories that correspond to the lower $33 \%$, middle $33 \%$ and upper $34 \%$ of 


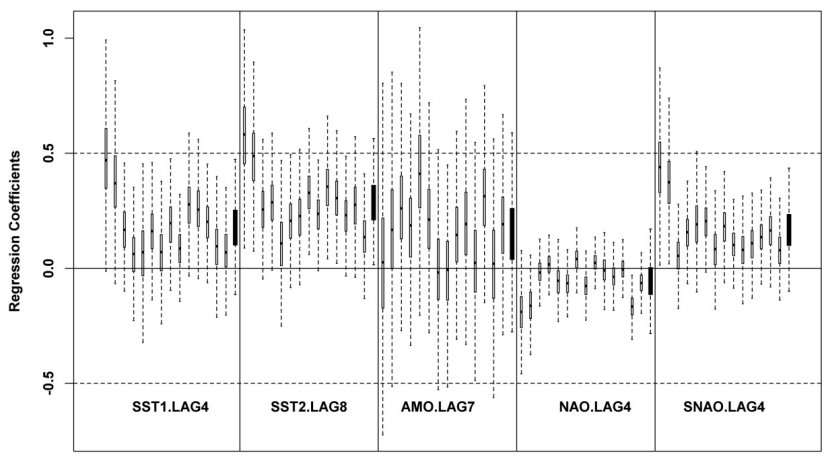

Climate Predictors for 14 Stations

Fig. 4. Box plots of the regression coefficients $\beta_{i j}$ (open box) and the $\boldsymbol{\mu}_{\beta j}$ mean of the regression coefficients (filled box) for the five predictors. The first two box plots for each predictor correspond to the streamflow stations and the next 12 to the rainfall stations.

values. These categories are determined separately for each station in the basin. Next, for each forecast at each station and year, the cumulative probability associated with each of the $\mathrm{k}$ categories is assessed by counting the fraction of the 1000 ensemble members from the hierarchical Bayesian regression model for that year for that station. Correspondingly, for each year and station, the observed cumulative probabilities for each category are assigned as 0 , if $\mathrm{k}<\mathrm{k}^{*}$, and 1 if $\mathrm{k} \geq \mathrm{k}^{*}$, where $\mathrm{k}^{*}$ is the category in which the observation for that year and station falls.

Now the RPS is computed as the squared difference between the observed and forecast cumulative probabilities, and the squared differences are summed over all three categories. The RPSS is then computed as

RPSS $=1-\frac{\overline{\mathrm{RPS}_{\text {forecast }}}}{\overline{\mathrm{RPS}_{\text {climatology }}}}$,

where $\overline{\mathrm{RPS}_{\text {forecast }}}$ is the mean ranked probability score for model forecast and $\overline{R P S}_{\text {climatology }}$ is the mean ranked probability score for climatological forecast. RPSS represents the level of improvement of the forecast in comparison to reference forecast which is usually assumed to be climatology. Similar to RE and CE, an RPSS $>0$ indicates that the forecasts have skill better that the climatology, and vice versa.

\section{Results and discussion}

The posterior distribution of the regression coefficients $(\boldsymbol{\beta})$ for each climate predictor and the mean of the vector of regression coefficients across sites $\left(\boldsymbol{\mu}_{\beta}\right)$ from the joint normal distribution, is shown in Fig. 4 through box plots of the values simulated from the posterior density functions. All the predictors except NAO have positive coefficients for the mean response across sites. Note that the spread on each $\boldsymbol{\mu}_{\beta}$ covers the median of the 14 corresponding $\beta \mathrm{s}$, as would be expected from partial pooling. With the exception of the AMO, the $\beta$ for streamflow tend to be higher and distinct from those for rainfall. This is expected given that the streamflow represents a spatial averaging of the rainfall process, and hence may have lower uncertainty and better identifiability. One could consider modeling these as separate groups to be pooled. However, given that we have only 2 streamflow stations, pooling across them would not provide much improvement in this application. Modeling them together provides a larger sample size (14) for the estimation of the coefficients of the Level 2 model, and leads to a higher spread in the posterior distribution of $\boldsymbol{\mu}_{\beta}$ than would result if we modeled the rainfall and streamflow stations in separate groups. An averaging of a larger number of rainfall stations with streamflow stations that individually represent a spatial averaging of rainfall is attractive from a conceptual perspective to regularize or reduce the uncertainty in the estimates of the response for the noisier rainfall stations. The simulations of the covariance matrix across predictors, $\boldsymbol{\Sigma}_{\beta}$ (results not shown), have non-zero off-diagonal elements. If two predictors are highly correlated then their regression coefficients cannot be uniquely identified through classical or Bayesian regression. However, their mean and covariance can be estimated and simulations of the $\log (\mathrm{Y})$ generated from the Bayesian model would be based on this covariance across the associated regression coefficients. Hence, an appropriate range of $\log (\mathrm{Y})$ values will be generated for each prediction, even though the individual $\beta$ are not uniquely identified due to predictor correlation.

The posterior probability distributions of the forecasts from the model for the streamflow at Bengbu station and rainfall at Shouxian station during the period 1996-2010 are shown as box plots in Fig. 5a and b, respectively. While the Bayesian model is developed using all the data, the forecasts are shown for the last 15 years to make a cleaner figure. Subsequent performance metrics are evaluated under cross-validation. The plots show streamflow and rainfall values as the percentage difference each year from their longterm average $\left(1696 \mathrm{~m}^{3} \mathrm{~s}^{-1}\right.$ for the flow at Bengbu station and $455 \mathrm{~mm}$ for rainfall at Shouxian). We see that the directional indication of the forecast is generally quite accurate while the uncertainty varies from year to year. We also computed the coverage rate under Bayesian credible intervals for the model and observe that for a $90 \%$ credible interval, on average, over the 14 stations, approximately $10 \%$ of the observed data are outside the interval, indicating the robustness of the fitted model.

From Fig. 5, we note that 1997, 1999 and 2001 are anomalously dry years, and that the observations are in the tails of the forecast probability distribution. First, we note that in terms of the usual practice in operational climate forecasting from NOAA or the IRI, where it is common to provide the users with a tercile forecast, the Bayesian model forecasts in each of these years do indicate a high probability of below normal values. Given that the forecast is developed using 4-6 

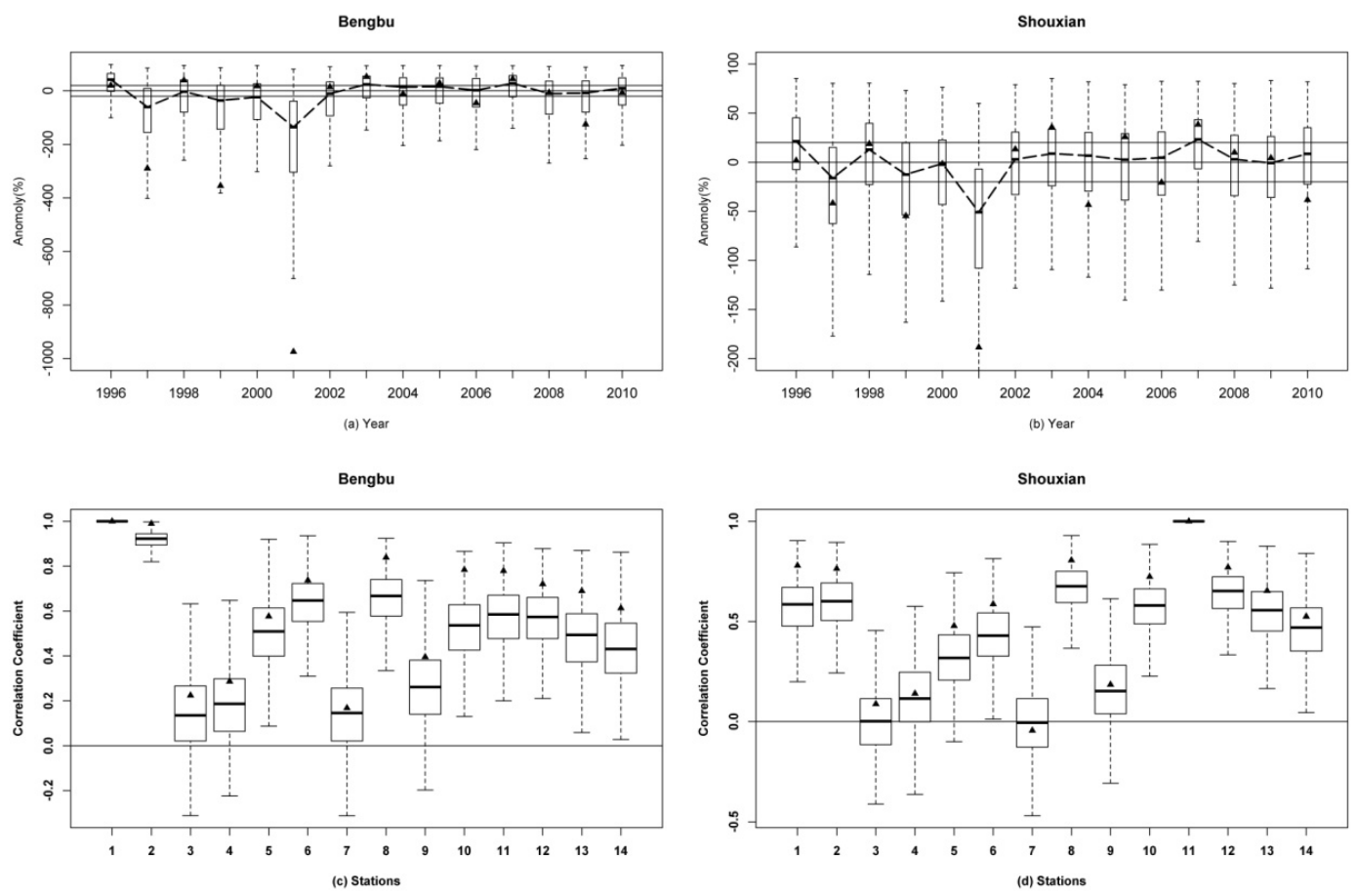

Fig. 5. The posterior probability distributions for JJA averaged streamflow and JJA total rainfall for (a) standardized streamflow at Bengbu station and (b) standardized rainfall at Shouxian station. The posterior distribution of the cross-site correlation for Bengbu station and for Shouxian station is presented in (c) and (d), respectively. Each box plot has the 25th, median and 75th percentile of the posterior distribution, with whiskers extended to the extreme values sampled. The solid triangle denotes the observation.

months lag predictors, the sign of a strong shift in the probability will alert the decision makers to an anticipated extreme event. Decision makers are often influenced by the ability to correctly indicate extreme conditions since the losses from their operations are most sensitive to such states. In our interactions with corporate and public sector decision makers, we have noticed both skepticism induced by failure to predict extremes, even if all performance measures are good, and conversely enthusiasm for the model on noting that the directional (high, average, low) forecasts are quite good.

However, we do see the consistent underestimation of dry conditions as evidence that either the tail behavior of the forecast distribution or the linearity of the link function between the predictors and the predictands is in question. The usual tests of goodness of fit accepted the hypothesis that the log-normal distribution was a good fit to the data, but discrimination with other distributions given the sample size may well lack power. Given the relatively short records, especially with our emphasis on cross-validation, and the dimension of the predictor space, exploration of a nonlinear model across the five predictors and 14 sites, and in the general case with more sites and predictors is challenging. In upcoming work, we are considering Gaussian process models (Rasmussen and William, 2006; Brahim-Belhouari and Bermak, 2004) to address this setting in a formal way.
To provide insight as to how applications of the categorical forecasts could be approached, we present (Fig. 6) the receiver/relative operating characteristic (ROC) plot (Mason, 1982) considering the decile categorical thresholds on the forecast posterior probability distribution for each of the 14 forecasts. Forecasts with better discrimination from random chance typically exhibit a ROC curve approaching the upperleft corner of the diagram as opposed to the $45^{\circ}$ diagonal lines, where the forecast has little ability to discriminate from a 50:50 probability that occurs by chance. From Fig. 6, we see that the ROC curve for all the 14 stations is well beyond the diagonal line and approaches the left corner indicating that the forecasts exhibit hit rates higher than the false alarm rate and are well calibrated to predict anomalous events using exogenous climate precursors. In reality a decision maker could prescribe their own thresholds of interest and evaluate the consequences of the forecast relative to the uncertainty and the threshold prescribed, as part of the decision process. In summary, while the conventional model checking under Bayesian modeling involves verifying for coverage rates and uncertainty level, we also verified our models using the standard verification procedures used in climate forecasting from various institutions for benchmarking (Barnston et al., 2003; Goddard and Mason, 2003).

It is important to have the proper spatial correlation of the forecasted streamflow across the stations for hydrologic 


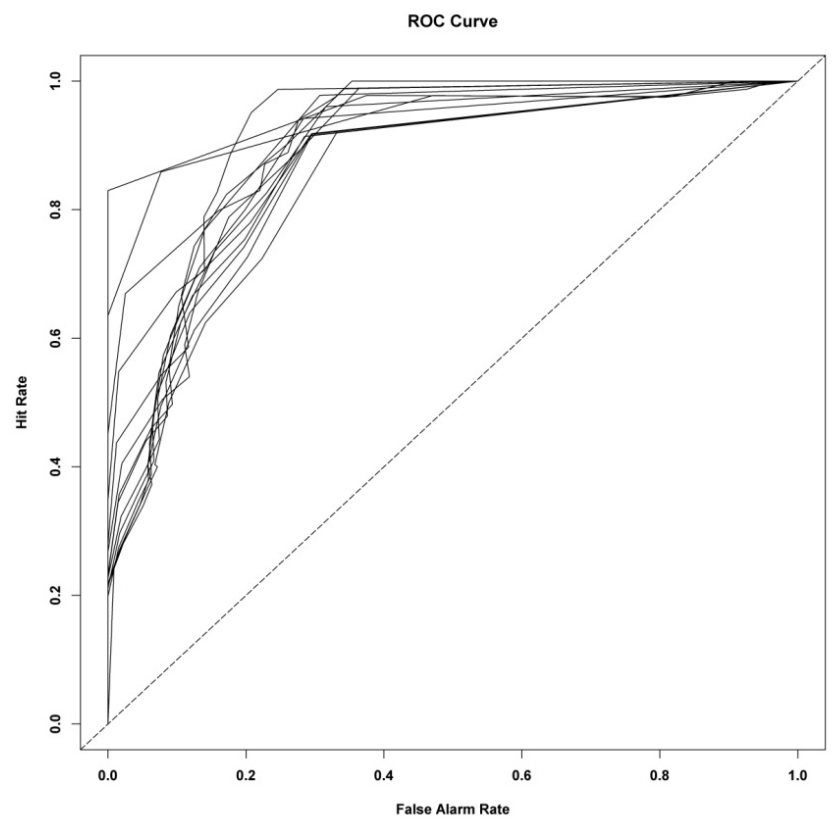

Fig. 6. ROC plot from the forecasts based on decile categorical thresholds for all the 14 streamflow and rainfall stations.

applications. Here, the spatial correlation is estimated from the posterior distribution of the streamflow and rainfall and compared to the observed cross-site correlation. The results for two stations (station1: streamflow from Bengbu station, station 11: rainfall from Shouxian station) are shown in Fig. 5c and d. The box plots in Fig. 5c and d present the posterior probability distribution of the correlations for these station with each of the 13 other stations. The observed correlation for each station is shown as a triangle. The forecast ensembles provide a good reproduction of the spatial correlation across all years.

The results for RE, CE, and RPSS performance under mfold cross-validation for each station are shown in Fig. 7. RE and CE are used to measure the goodness of fit of the model by comparing the forecasted streamflow and rainfall with the actual observed data. They are used as an expression of the true $R^{2}$ of the regression equation when applied to new data. By assessing the RE and CE under cross-validation, we are essentially providing a measure of the variance explained under a validation data set. Given that seasonal forecasts are better represented probabilistically using the posterior distribution, expressing the skill of the forecast using RE and $\mathrm{CE}$ requires summarizing the forecasts using measures of central tendency such as mean or median of the posterior distribution which does not give credit to the probabilistic information in the forecasts. RPSS computes the cumulative squared error between the categorical forecast probabilities and the observed category in relevance to a reference forecast. We observe that typically the hierarchical Bayesian model leads to values of RE, CE and RPSS greater than zero
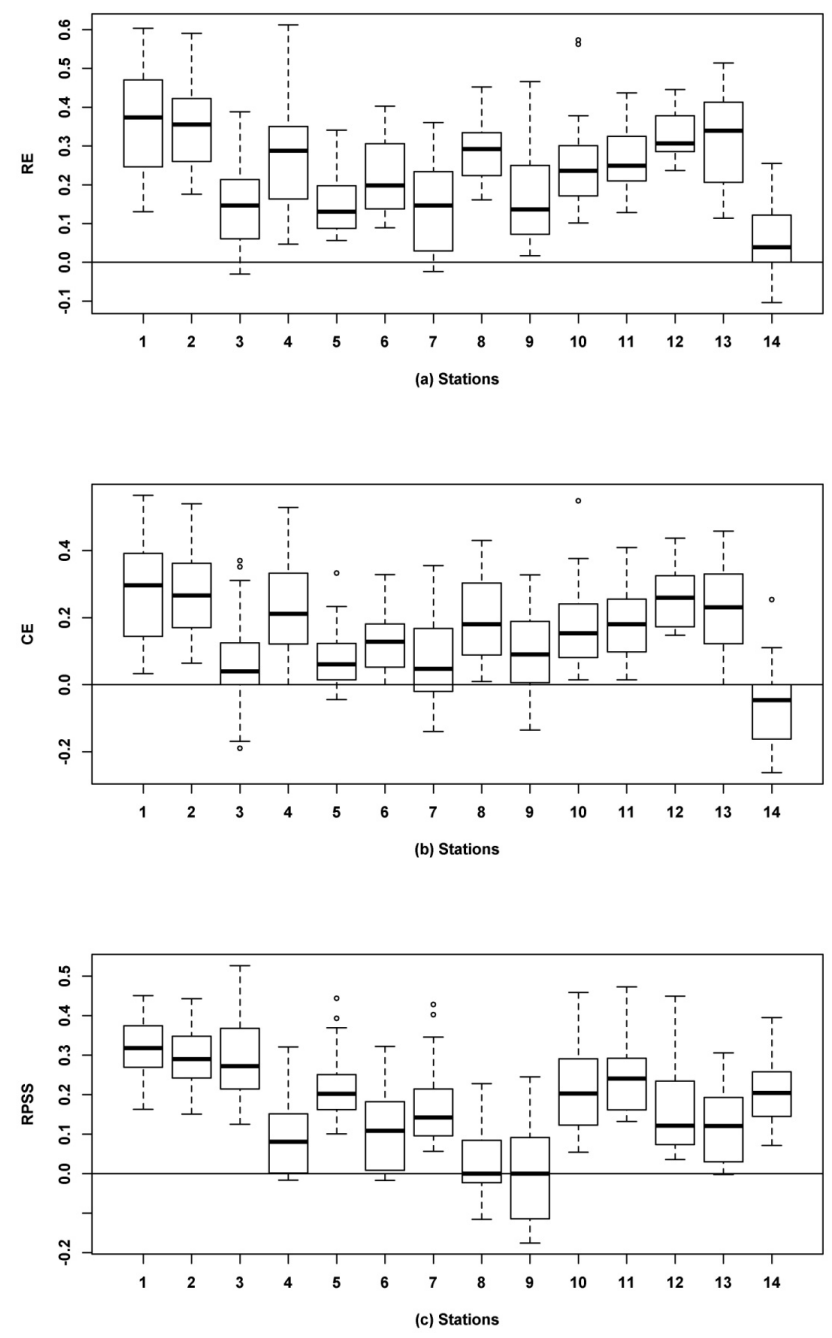

Fig. 7. Performance under "leave-10-out cross-validation" for all 14 stations from the hierarchical Bayesian model from 30 random simulations - (a) reduction of error (RE), (b) coefficient of efficiency (CE), (c) Rank probability skill score (RPSS).

for all the stations (except Huoshan station -14) indicating that the seasonal forecasts developed using the climate precursors contain useful information.

In addition to computing these traditional performance measures, the performance of the posterior probability distribution is assessed by examining the model's ability to cover the observed rainfall and flows within a specified credible interval under $\mathrm{m}$-fold cross-validation. We estimated the coverage rates for the $90 \%$ credible intervals under crossvalidation. For each validation period, we count the number of failures or the number of observations that are outside the 5th and 95th percentile of the posterior distribution for each station resulting from the model developed using the fitting period. By computing the total number of failures from all the randomly selected models, we estimated the coverage rate as the percentage of failures for a total of 300 
$(30 \times 10$ years $)$ forecasts. The average coverage rate across the stations is $92 \%$ for the corresponding to the $90 \%$ coverage interval indicating the robustness of the fitted Bayesian models under cross-validation.

\section{Summary}

This study investigated the predictability of the summer rainfall and streamflow in the upper and mid-Huai River basin using five selected large-scale climate indices as predictors. The study identified two regions, one in the North Pacific and one in the North Atlantic in addition to pre-season AMO, NAO and SNAO that can be used as climatic precursors for JJA streamflow and rainfall in the Huai River basin. As the underlying challenge is to consistently model the variability across sites and across variables, we employed a hierarchical Bayesian model strategy. Hierarchical Bayesian models and multi-level models have become quite common as educational and research tools for Computational Statistics. They appear for applications in causal inference, prediction, comparison and data description especially for multi-variable problems where the investigator needs to learn something about the group as well as individual dynamics. In many cases, they directly generalize traditional regression approaches in this setting. Given our experience with such models in other contexts, we were interested in exploring how a structured approach to regional statistical forecasts of streamflow and rainfall could be approached. In general, this is a high dimensional problem that offers some interesting opportunities both from a model framing context and from a regularization context.

The partial-pooling hierarchical Bayesian regression model provided a useful way to model spatial co-variability in seasonal hydrological predictions, while considering the potentially common effects of the predictors on regional hydrologic response. An advantage of the approach is that it allows appropriate grouping of information in the region, and explicit modeling of the covariance of the model errors and the regression coefficients to better represent the uncertainty in both the model parameters and the final streamflow and rainfall forecasts. Cross-validated model results show good predictive skill, and the common effects as well as the at site effects of each predictor are identified well even under leave-10-out of 50 cross-validation. Comparison of the partial-pooling model with a no-pooling model in the same estimation framework (equivalent to the traditional regression based modeling framework) showed that the Bayesian model is competitive or superior in terms of the validation statistics. An aspect of Bayesian modeling that is often cited is the ability to provide a systematic approach to the propagation and modeling of model parameter uncertainty. In this context, we argue that while there could be a potential upper limit in predictability in seasonal rainfall using anomalous SST conditions as shown in Westra and Sharma (2010), from a forecast utility point of view, knowing the uncertainty is certainly useful since it can be used for developing probability-based risk management models for optimizing reservoir operations or agricultural decision models. We find this useful, but also note that typically these models require assumptions as to parametric probability density models. There is a practical utility especially in the context of dynamical and statistical regional forecast models to being able to generate consistent simulations across parameters and output variables and to build in a multi-level modeling structure in one shot using multi-level or hierarchical models. Our future work in the region will focus on using the forecasts developed to specify dynamic rules for operating multiple reservoir systems in the basin using both multi-site seasonal hydrologic forecasts and changing demand through adaptive human behavior to better manage deficits from the reservoirs. A refinement of the method applied here to disaggregating the rain and streamflow in time over the season to properly capture monsoon breaks and the amplitude, duration and spatial structure of rainfall events will be a goal for these applications. Nonlinearity and non-Gaussian aspects will be modeled in a Gaussian process framework.

Acknowledgements. We thank the Royal Netherlands Meteorological Institute (KNMI), hydrological bureau of Huai River and China Meteorological Data Sharing Service System for the data used in this study. The work was funded in part by China Scholarship Council, the PepsiCo Foundation, the Columbia Water Center, the National Basic Research Program of China (grant no. 2010CB951103), Nonprofit Industry Research Special Fund of China's MWR (201101003), National Natural Science Foundation of China (41101015, 41271042 and 41101016), the Special Fund of State Key Laboratory of Hydrology-Water Resources and Hydraulic Engineering (1069-50985512), the "Strategic Priority Research Program" of the Chinese Academy of Sciences XDA05110102. The authors would like to thank the two anonymous reviewers whose valuable comments led to significant improvements in the manuscript.

Edited by: H. H. G. Savenije

\section{References}

Barnston, A. G., Mason, S. J., Goddard, L., Dewitt, D. G., and Zebiak, S. E.: Multimodel ensembling in seasonal climate forecasting at IRI, B. Am. Meteorol. Soc., 84, 1783-1796, 2003.

Brahim-Belhouari, S. and Bermak, A.: Gaussian process for nonstationary time series prediction. Comput. Stat. Data Anal., 47, 705-712, 2004.

Candille, G. and Talagrand, O.: Evaluation of probabilistic prediction systems for a scalar variable, Q. J. Roy. Meteorol. Soc., 131, 2131-2150, doi:10.1256/qj.04.71, 2005.

Cheng, Z., Xu, M., Luo, L. S., and Ding, X. J.: Climate characteristics of drought-flood abrupt change events in Huai River basin, J. China Hydrol., 32, 73-79, 2012 (in Chinese). 
Devineni, N., Lall, U., Pederson, N., and Cook, E.: A Tree Ring based Reconstruction of Delaware River Basin Streamflow using Hierarchical Bayesian Regression, J. Climate, 26, 4357-4374, doi:10.1175/JCLI-D-11-00675.1, 2013.

Fritts, H. C.: Tree rings and climate, Academic Press, London, 1976.

Folland, C. K., Knight, J., Linderholm, H. W., Fereday, D., Ineson, S., and Hurrell, J. W.: The summer North Atlantic Oscillation: past, present, and future, J. Climate, 22, 1082-1103, 2009.

Gangopadhyay, S., Clark, M., and Rajagopalan, B.: Statistical downscaling using K-nearest neighbors, Water Resour. Res., 41, W02024, doi:10.1029/2004WR003444, 2005.

Gelman, A. and Hill, J.: Data Analysis Using Regression and Multilevel/Hierarchical Models, Cambridge University Press, New York, NY, 2007.

Geng, Q. Z., Ding, Y. H., and Huang, C. Y.: Influences of the Extra tropical Pacific SST On the Precipitation of the North China Region, Adv. Atmos. Sci., 14, 339-349, 1997.

Goddard, L. and Mason, S. J.: Evaluation of the IRI's "net assessment" seasonal climate forecasts: 1997-2001, B. Am. Meteorol. Soc., 84, 1761-1781, 2003.

Gu, W., Li, C. Y., Wang, X., and Zhou, W.: Linkage between mei-yu precipitation and North Atlantic SST on the decadal timescale, Adv. Atmos. Sci., 26, 101-108, doi:10.1007/s00376-009-01015, 2009a.

Gu, W., Li, C. Y., Wang, X., Zhou, W., and Chan, J. C. L.: Interdecadal unstationary relationship between NAO and east China's summer precipitation patterns, Geophys. Res. Lett., 36, L13702, doi:10.1029/2009GL038843, 2009b.

Hurrell, J. W., Kushnir, Y., Ottersen, G., and Visbeck, M. (Eds.): The North Atlantic Oscillation: Climatic Significance and Environmental Impact, Geophys. Monogr. Ser., Vol. 134, 279 pp., AGU, Washington, D. C., doi:10.1029/GM134, 2003.

Knight, J. R., Folland, C. K., and Scaife, A. A.: Climate impacts of the Atlantic Multidecadal Oscillation, Geophys. Res. Lett., 33, L17706, doi:10.1029/2006GL026242, 2006.

Kwon, H.-H., Brown, C., and Lall, U.: Climate informed flood frequency analysis and prediction in Montana using hierarchical Bayesian modeling, Geophys. Res. Lett., 35, L05404, doi:10.1029/2007GL032220, 2008.

Kwon, H.-H., Brown, C., Xu, K., and Lall, U.: Seasonal and annual maximum streamflow forecasting using climate information: Application to the Three Gorges Dam in the Yangtze River basin, China, Hydrol. Sci. J., 54, 582-595, 2009.

Lima, C. H. and Lall, U.: Hierarchical Bayesian modeling of multisite daily rainfall occurrence: Rainy season onset, peak, and end, Water Resour. Res., 45, W07422, doi:10.1029/2008WR007485, 2009.

Lima, C. H. and Lall, U.: Spatial scaling in a changing climate: A hierarchical bayesian model for non-stationary multi-site annual maximum and monthly streamflow, J. Hydrol., 383, 307$318,2010$.
Linderholm, H. W., Ou, T., Jeong, J.-H., Folland, C. K., Gong, D., Liu, H., Liu, Y., and Chen, D.: Interannual teleconnections between the summer North Atlantic Oscillation and the East Asian summer monsoon, J. Geophys. Res., 116, D13107, doi:10.1029/2010JD015235, 2011.

Lorenz, E. N.: Empirical orthogonal functions and statistical weather prediction. MIT statistical forecasting project report no.1, contract AF 19, 604-1566, 1956.

Lu, R., Dong, B., and Ding, H.: Impact of the Atlantic Multidecadal Oscillation on the Asian summer monsoon, Geophys. Res. Lett., 33, L24701, doi:10.1029/2006GL027655, 2006.

Mason, I.: A model for assessment of weather forecasts, Aust. Met. Mag., 30, 291-303, 1982.

Rasmussen, C. E. and William, C. K. I.: Gaussian processes for machine learning, MIT Press, 2006.

Rayner, N. A., Brohan, P., Parker, D. E., Folland, C. K., Kennedy, J. J., Vanicek, M., Ansell, T. J., and Tett, S. F. B.: Improved Analyses of Changes and Uncertainties in Sea Surface Temperature Measured In Situ since the Mid-Nineteenth Century: The HadSST2 Dataset, J. Climate, 19, 446-469, doi:10.1175/JCLI3637.1, 2006.

Renard, B., Sun, X., and Lang, M.: Bayesian Methods for Nonstationary Extreme Value Analysis, in: Extremes in a Changing Climate, 39-95, Springer Netherlands, 2013.

Robertson, A. W., Kirshner, S., and Smyth, P.: Downscaling of daily rainfall occurrence over northeast Brazil using a hidden Markov model, J. Climate, 17, 4407-4424, 2004.

Souza Filho, F. A. and Lall U.: Seasonal to interannual ensemble streamflow forecasts for Ceara, Brazil: Applications of a multivariate, semiparametric algorithm, Water Resour. Res., 39, 13071319, doi:10.1029/2002WR001373, 2003.

Spiegelhalter, D., Thomas, A., Best, N., and Gilks, W.: BUGS 0.5: Bayesian inference Using Gibbs Sampling-Manual (version ii), Medical Research Council Biostatistics Unit, Cambridge, 1996.

Westra, S. and Sharma, A.: An Upper Limit to Seasonal Rainfall Predictability?, J. Climate, 23, 3332-3351, doi:10.1175/2010JCLI3212.1, 2010.

Wilks, D. S.: Statistical methods in the atmospheric sciences, Access Online via Elsevier, 2011.

Xu, K., Brown, C., Kwon, H. H., Lall, U., Zhang, J., Hayashi, S., and Chen, Z.: Climate teleconnections to Yangtze river seasonal streamflow at the Three Gorges Dam, China, Int. J. Climatol., 27, 771-780, doi:10.1002/joc.1437, 2007.

Yan, D. H., Wu, D., Huang, R., Wang, L. N., and Yang, G. Y.: Drought evolution characteristics and precipitation intensity changes during alternating dry-wet changes in the HuangHuai-Hai River basin, Hydrol. Earth Syst. Sci., 17, 2859-2871, doi:10.5194/hess-17-2859-2013, 2013.

Zhang, Y., Arthington, A. H., Bunn, S. E., Mackay, S., Xia, J., and Kennard, M.: Classification of flow regimes for environmental flow assessment in regulated rivers: the Huai River Basin, China, River Res. Applic., 28, 989-1005, doi:10.1002/rra.1483, 2012. 